\title{
MECHATRONIC SCANNING SYSTEM WITH INTEGRATED MICRO ELECTRO MECHANICAL SYSTEM POSITION SENSORS*
}

\author{
VLADimir STAVRov \\ AMG Technology Ltd., Botevgrad, Bulgaria \\ e-mail: vs@amg-t.com \\ Dimitar Chakarov, Assen Shulev, Mihail Tsveov \\ Institute of Mechanics, Bulgarian Academy of Sciences, \\ Acad. G. Bonchev St., Bl. 4, 1113 Sofia, Bulgaria \\ e-mails:mit@imbm.bas.bg, assenshulev@gmail.com, mtsveov@abv.bg
}

[Received 11 May 2016. Accepted 20 June 2016]

\begin{abstract}
In this paper, a study of a mechatronic scanning system for application in the microbiology, microelectronics research, chemistry, etc. is presented. Integrated silicon micro electro mechanical system (MEMS) position sensor is used for monitoring the displacement of the scanning system. The utilized silicon MEMS sensors with sidewall embedded piezoresistors possess a number of key advantages such as high sensitivity, low noise and extremely low temperature dependence. Design of $2 \mathrm{D}$ scanning system with a travel range of $22 \times 22 \mu \mathrm{m}^{2}$ has been presented in present work. This system includes a Compliant Transmission Mechanism, (CTM) designed as a complex elastic mechanism, comprising four parallelograms. Computer aided desigh (CAD) model and finite element analysis (FEA) of the Compliant Transmission Mechanism mechanisms have been carried out. A prototype of the scanning system is fabricated, based on CAD model. An experimental set-up of an optical system and a correlation technique for digital image processing have been used for testing the scanning system prototype. Results of the experimental investigations of the prototyped scanning system are also presented. KEY WORDS: Scanning system, micro electro mechanical system (MEMS) sensor, piezoresistors, transmission mechanism, finite element analysis (FEA) simulations, experimental set-up, digital image processing.
\end{abstract}

\footnotetext{
${ }^{*}$ Corresponding author e-mail: mit@imbm.bas.bg.

We gratefully acknowledge the financial supports of this work by grant 6IF-0213/15.12.2012 of the Bulgarian National Innovation Fund.
} 


\section{Introduction}

Many micro- and nano-processes have been automated, facilitating the access to the micro- and nano-domains with unknown dynamics and properties. At the same time, the mechatronic approach to these processes creates multiple problems to be resolved. One of them is the development of mechanical structures appropriate for monitoring and control of working tools to be applied in the domains of microsurgery and microbiology, microelectronics research, chemistry and investigation of thin films, scanning of micro- and nano-surfaces, micro assembly etc. [1, 2, 3].

These devices comprise high-precision mechanical structures free of backlash, friction and hysteresis [2], in order to obtain the required submicron accuracy. Mechanisms with closed kinematics chains are suitable for high-precision applications $[4,5,6]$. As it is well known, parallel kinematics mechanisms possess inherent advantages over mechanisms with open kinematics chains, such as high: rigidity, load capacity, velocity, precision, etc.

On the other hand, compliant mechanisms, i. e., flexure-based mechanisms can be employed into parallel mechanisms for high-precision applications $[4,7,8]$. Compliant mechanisms exhibit many advantages in terms of vacuum compatibility: they have neither backlash behaviour nor nonlinear friction and they are simple and easy to fabricate. The use of a compliant mechanism, in order to provide motion transfer, means that the position accuracy of such micromotion mechanisms depends only on the accuracy of micro-actuators and on the position sensors.

Various actuation principles and actuators have been exploited to drive the compliant mechanism in the scanning devices - piezoelectric, electrostatic, electromagnetic, and shape memory alloy actuators. They have been utilized to provide fine motions of the micro- and nano-devices. Piezoelectric actuators are commonly used to ensure fine resolution of input displacements in the sub-nanometre range $[3,9,10,11]$, since their resolution depends solely on the applied voltage signal. For example, piezoelectric actuators with closed kinematical structures are suitable for high-precision 3D applications [6, 12].

Various approaches exist for assessment of stress and strain in elastic systems. One of them is the so-called Finite Element Analysis (FEA) [4, 7, 13]. The FEA method has been proven to be the most accurate and reliable one, since the joints are represented with their real sizes and shapes.

The progress in the area of Micro Electro Mechanical Systems (MEMS) has broadened considerably the applications of these devices. MEMS technol- 
ogy has also enabled the miniaturization of the devices and a typical MEMS sensor is at least one order of magnitude smaller, as compared to a conventional metal-foil strain sensor, that is used to measure the same quantity. Consequently, MEMS devices can be batch-fabricated, which offers a high potential for cost reduction. Moreover, proper design of the devices can resolve problems, related to power consumption, while providing improved performance characteristics, such as accuracy, low thermal sensitivity and resolution [14].

Several physical sensing principles have been explored in MEMS strain sensors, including the modulation of optical, capacitive, piezoelectric, and piezoresistive properties or frequency shift $[15,16]$. More particularly, piezoresistive MEMS strain sensors are favourable and attractive, due to a number of key advantages such as high sensitivity [17], low noise, better scaling characteristics, low cost and their ability to have the detection electronics circuit further away from the sensor, or on the same sensing board. Moreover, they have high potential for monolithic integration with low-power CMOS electronics and piezoresistive strain sensors and need less complicated conditioning circuits.

In this paper, a study of a scanning system with integrated silicon MEMS position sensor with sidewall piezoresistors and the design of a $2 \mathrm{D}$ scanning system with a travel range of $22 \times 22 \mu \mathrm{m}^{2}$, as well as CAD model and FEA simulations of the elastic transmission mechanisms and results of the experimental investigations of the prototyped scanning system are presented.

\section{MEMS position sensor for detection of $500 \mu \mathrm{m}$ range dis-}

\section{placement}

MEMS position sensor is chosen for displacement detection in the scanning system. Particularly, a contact MEMS device with a travel range of 500 $\mu \mathrm{m}$ has been used for displacement detection [18]. The envisaged position micro-sensor comprises of a single anchored (1) and a single moveable (2) part. Both parts are connected with a monolithic flexure (3), shown in the Fig. 1(a). The monolithic flexure (3) comprises two pairs of differential springs and two detecting cantilevers (4), which are also attached at the point $C$ of spring's connection.

The cantilevers are oriented in X direction and the move of part (2) in $\mathrm{Y}$ direction is transduced to a bending of cantilevers (4) by differential springs. In the sidewalls of the fixed end of the both cantilevers (4) four piezoresistors are embedded $\left(5_{1} \div 5_{4}\right)$. In more details, each cantilever possesses a pair of two piezoresistors, $5_{1}-5_{2}$ and $5_{3}-5_{4}$, respectively, which are electrically connected 


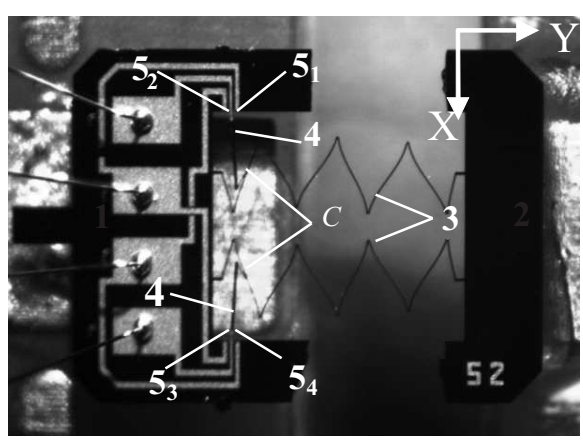

(a)

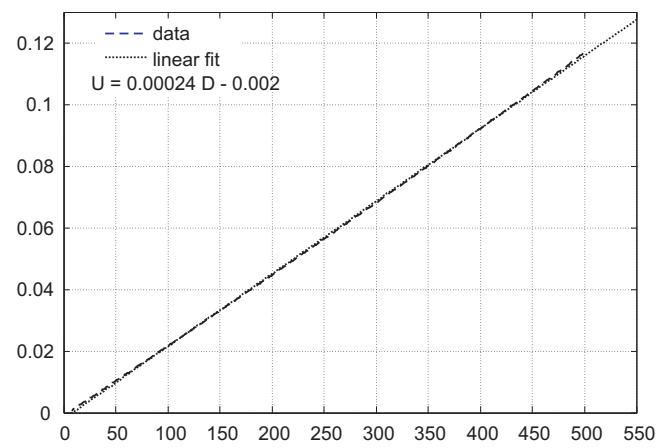

(b)

Fig. 1. (a) Optical micrograph of a position micro-sensor with a single anchored (1) and a single actuated (2) part and a flexure (3) comprising two cantilevers (4), oriented in $\mathrm{X}$ direction; four sidewall piezoresistors $\left(5_{1} \div 5_{4}\right)$, sensitive in $\mathrm{Y}$ direction are built-in at the fixed ends of the cantilevers; (b) Plot of the sensor signal, showing $240 \mu \mathrm{V} / \mu \mathrm{m}$ sensitivity and $500 \mu \mathrm{m}$ travel range

in two voltage dividers. Both voltage dividers are further so connected in a full bridge configuration, to amplify each other, when actuated part (2) moves in Y (horizontal) direction. This particular sensor has demonstrated a displacement sensitivity of $240 \mu \mathrm{V} / \mu \mathrm{m}$ and $1 \mathrm{~V}$ DC voltage supply of the bridge, as plotted in Fig. 1(b), and the travel range is limited to approx. $500 \mu \mathrm{m}$.

Additionally, the devices with sidewall embedded piezoresistors exhibit very low noise and extremely low (i.e., non-detected at all) temperature dependence [19]. The sensitivity and the travel range can be tuned to meet the optimization criteria [20], by means of a modification of the flexure layoutThis particular flexure (3) can be used in tensile mode only, since differential springs, displayed in Fig. 1(a), are mechanically unstable in compression mode.

As far, the detecting cantilevers (4) with sidewall piezoresistors ensure sensor signal having above 1,000,000 of scale intervals at full scale range, there is a room to achieve a ppm (part per million) resolution of the position sensor. Since the silicon flexures are extremely fragile, auxiliary mechanical parts with package features for fixing and protecting both MEMS parts and providing a relative displacement in the range from $50 \mu \mathrm{m}$ to $1.5 \mathrm{~mm}$, have been developed. 


\section{Design of the scanning system}

The 2D scanning system has been developed with a travel range of $22 \times 22 \mu \mathrm{m}^{2}$, along axes of a Cartesian coordinate system 0XY. This system includes a Compliant Transmission Mechanism (CTM), designed as a complex parallelogram mechanism for displacements in the plane 0XY, as shown in Fig. $2(\mathrm{a})$.

The CTM incorporates four common parts marked as 1, 2, 3, 4 and interconnected by four parallelograms, according to Fig. 2(a). The main part 1 is anchored and the orientation of the moveable part 4 is kept constant. All joints in the CTM are shaped as double-notched elastic beam joints. These joints possess a series of advantages compared to contact ones, as they are free from backlash, friction, and hysteresis. They allow, as well, pre-stressing of the actuators by means of a providing preliminary stress.

Two mutually orthogonal actuators A1 and A2 are placed between the first anchored part 1 and both movable corner parts 2 and 3 (Fig. 2(b)). Piezoelectric stack actuators of Physik Instrumente (PI) GmbH \& Co. KG have been chosen as actuators of the device, hereafter called piezoactuators, because of their smooth motion, high accuracy, and quick response. The parameters of the exploited piezoactuators are shown in Table 1.

By controlling the position of the both piezo actuators $A_{1}$ and $A_{2}$, the mechanism is elastically stressed to scan the fourth_part along axes $0 \mathrm{X}$ and $0 \mathrm{Y}$, respectively within $22 \times 22 \mu \mathrm{m}^{2}$ in the plane $0 \mathrm{XY}$. The CTM is shielded on

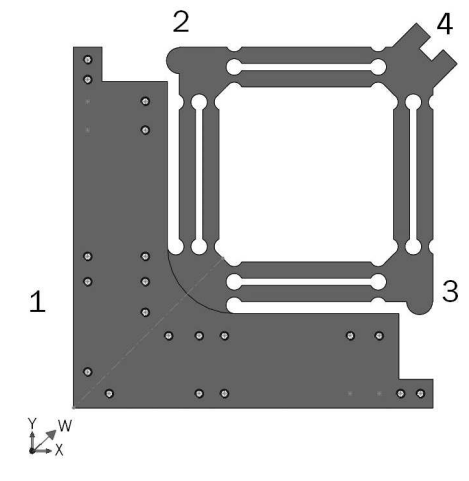

(a)

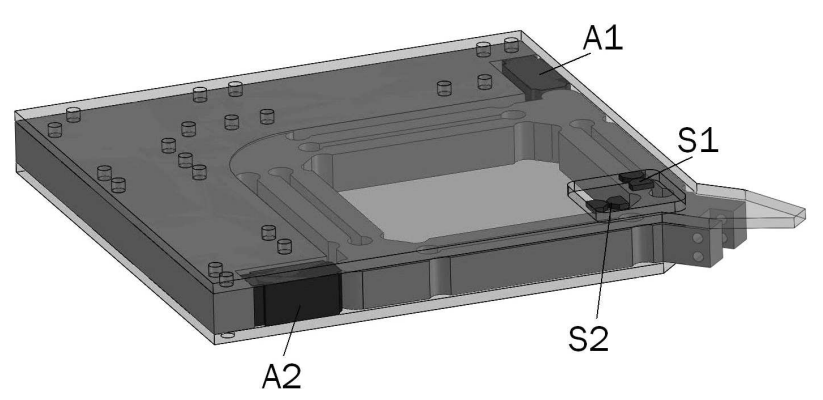

(b)

Fig. 2. CAD model of the: (a) compliant transmissions mechanism (CTM); (b) scanning system with two piezoactuators A1, A2 and two MEMS sensors S1, S2 
Table 1. Parameters of the exploited actuators

\begin{tabular}{|c|c|c|c|c|c|c|}
\hline Actuator & Type & $\begin{array}{c}\text { Travel } \\
{[\mu \mathrm{m}]}\end{array}$ & $\begin{array}{c}\text { Resonant } \\
\text { frequency } \\
{[\mathrm{kHz}]}\end{array}$ & $\begin{array}{c}\text { Axial } \\
\text { stiffness } \\
{[\mathrm{N} / \mu \mathrm{m}]}\end{array}$ & $\begin{array}{c}\text { Blocking } \\
\text { force } \\
{[\mathrm{N}]}\end{array}$ & $\begin{array}{c}\text { Dimensions } \\
\mathrm{A} \times \mathrm{B} \times \mathrm{L} \\
{[\mathrm{mm}]}\end{array}$ \\
\hline $\mathrm{A} 1, \mathrm{~A} 2$ & $\mathrm{P}-885.51$ & 18 & 70 & 50 & 900 & $5 \times 5 \times 18$ \\
\hline
\end{tabular}

both flat sides by upper and lower thin plates, attached to the stationary part 1 and keeping a small gap between the plates and the stressed parts as shown in Fig. 2(b).

MEMS piezoresistive strain sensors are used to measure the displacements in the device and to generate a position feedback signal. Furthermore, each sensor monitors the displacement along one axis and allows deviations in the other axes. Two MEMS sensors S1 and S2 are used to monitor the displacements for axes 0X, 0Y. All sensors are composed of a base part and a moving part. The base parts of the sensors S1 and S2 are located on the upper shield plate. The moving parts of the sensors S1 and S2 are attached to the fourth body of the CTM, as it is shown in Fig. 2(b). The sensors are mounted orthogonally relative to one another. In this way, the two MEMS sensors measure the displacements of the end effector of the scanning system in two axes system OXY independently.

A pre-stressing of the mechanical system is provided, in order to eliminate the backlash and improve the performance of the piezoactuators. Prestressing is carried out by means of an assembly offset along the axes of the piezoactuators, which lead to deflection in all the system elastic joints and stressing of the actuators by restoring forces. Assembly offset in the range of $20-100 \mu \mathrm{m}$ is performed through negative clearances in actuator beds.

Based on the CAD model, presented in Fig. 2(b), a prototype of the CTM is fabricated. The compliant transmission mechanism is manufactured of stainless steel X2CrNi18-9 (on DIN) with Young modulus $E=200 \mathrm{GPa}$, tensile strength $\sigma_{t}=0.600 \mathrm{GPa}$ and yield strength $\sigma_{y}=0.400 \mathrm{GPa}$. The mechanism is accomplished with the help of electro discharge machining of monolithic block with thickness $8 \mathrm{~mm}$.

\section{FEA simulations}

An experiment of the static load of the CTM was carried out, using FEA simulations as an embedded part of the CAD system. The simulations were conducted at forces, applied at contact point between actuator and moving 
part of the mechanism. The system body is simulated by means of the presented above material - stainless steel, and the stack piezo-actuators are modelled as linear elastic joints, possessing an axial stiffness $50.10^{6}[\mathrm{~N} / \mathrm{m}]$.

Simulations were conducted, when stressing forces of $100 \mathrm{~N}$ are attached at the point of application of first actuator A1 and at the point of application of second actuator A2. The screenshot of the effective displacements is shown in Fig. 3(a). Effective displacements of the end effector are $59 \mu \mathrm{m}$ along $\mathrm{X}$ direction and $61.6 \mu \mathrm{m}$ along $\mathrm{Y}$ direction. These are displacement values for pre-stressed mechanism. Yielding stresses in the loading area do not exceed the admissible value for the material of $\sigma_{y}=0.400$ [GPa].

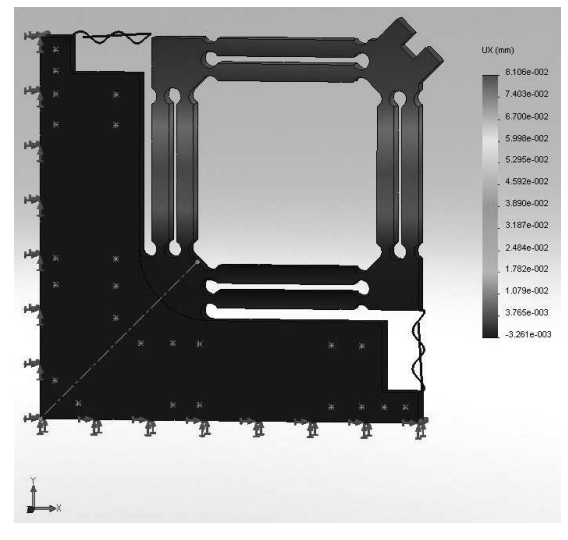

(a)

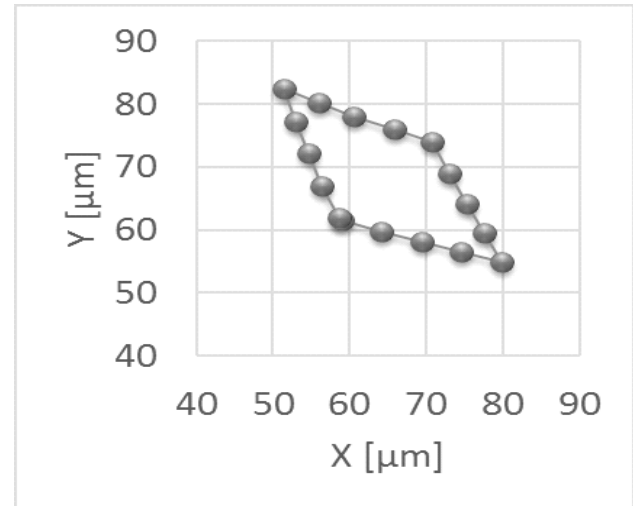

(b)

Fig. 3. FEA simulations of the CTM: (a) screens with effective displacements along the $\mathrm{X}$ axis; (b) graph of the end effector displacements in OXY plane

Furthermore, the force of actuator A1 is increased with uniform steps from $100 \mathrm{~N}$ to $120 \mathrm{~N}$, then the force of the second actuator A2 is increased evenly from $100 \mathrm{~N}$ to $120 \mathrm{~N}$, after which the force of the first actuator decreases to $100 \mathrm{~N}$ and finally, decreases the force of the second actuator to $100 \mathrm{~N}$. The resulting displacement of the end effector is recorded at each step. Effective displacements in the point of application of an actuator reach $18 \mu \mathrm{m}$, which is the limit of the piezoactuator travel range. As a result, the graph of the end-effector position in OXY plane is illustrated in Fig. 3 (b).

The figure shows the travel range in $0 \mathrm{XY}$ plane of scanning device. As can be seen from Fig. 3(b), when an actuator performs a move of $18 \mu \mathrm{m}$, the CTM end effector achieves a move along one axis of $21 \mu \mathrm{m}$ and an offset along 
the other axis of $8 \mu \mathrm{m}$. This experiment shows the cross-influences between axes. This motion coupling must be taken into account, when control the scanning device.

\section{Experimental set-up for optical investigation of the scanning} system

A prototype of the scanning system, based on the developed CAD model, was fabricated. The assembled scanning system consists of CTM (Fig. 4(a)), upper and lower thin plates, piezoactuators A1, A2, as well as MEMS S1 and S2 (Fig. 4(b)).

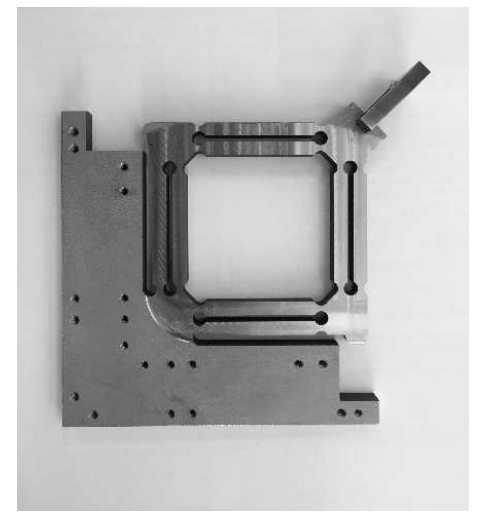

(a)

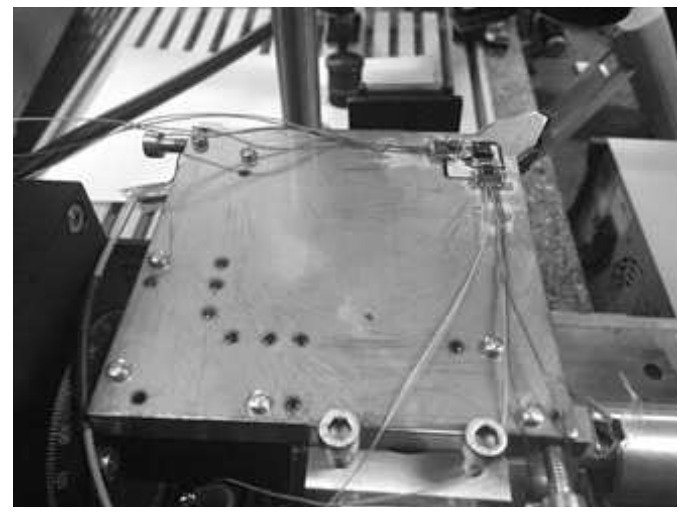

(b)

Fig. 4. Pictures of the scanning system prototype: a) the elastic transmission mechanism; b) the assembled system

The experimental set-up comprises a Computer Vision System (CVS), a precise translational stage, and the prototyped scanning system mounted on it (Fig. 5). The translational stage was used for precise alignment of the scanning system with respect to the vision system, and for image space calibration. It was constructed from three orthogonal translational modules with integrated linear positioning sensors with a resolution of $0.1 \mu \mathrm{m}$. All modules were actuated by step-motors, controlled via a 3-channel control system connected to a PC.

The end-effector movements of the Scanning System prototype have been investigated by means of the Computer Vision System, using displacement measurement techniques, based on a Digital Image Correlation method [21]. They allow estimating the end-effector's displacements in the image plane 


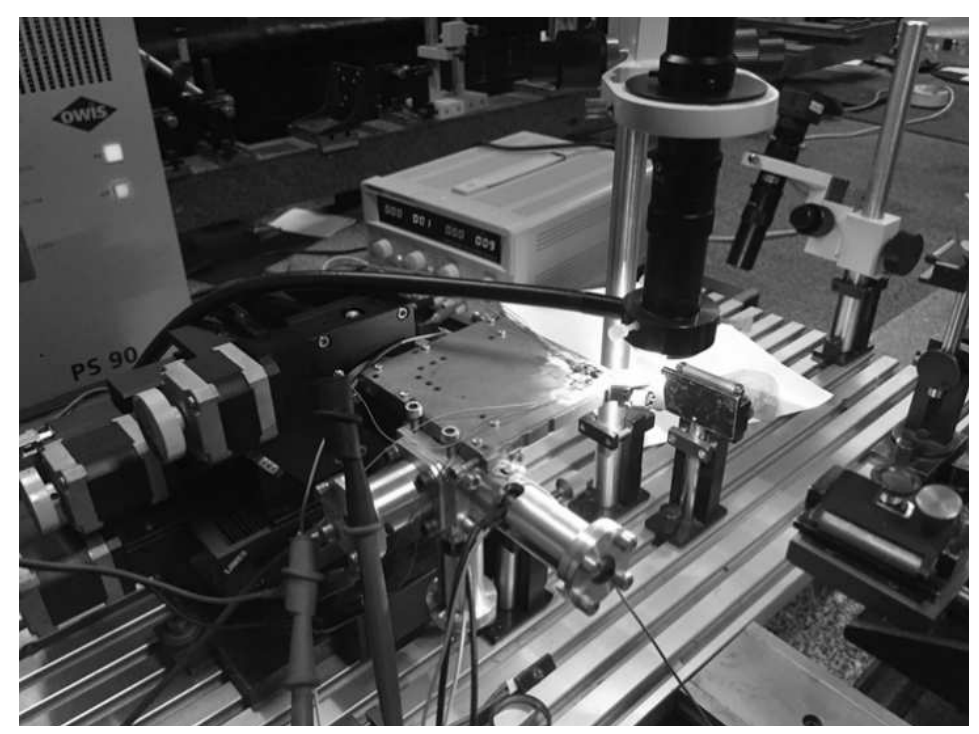

Fig. 5. Picture of the experimental set-up

between two successive images. Therefore, knowing the optical magnification and pixel dimensions could provide also the real displacements magnitude.

This CVS consists of a high quality zoom-imaging lens with a magnification of $2.5 \mathrm{x}-10 \mathrm{x}$ at a constant working distance of $35 \mathrm{~mm}$, CMOS camera with $2592 \times 1944$ pixels, each of them with dimensions $2.2 \times 2.2 \mu \mathrm{m}^{2}$ and a computer. The camera's frame rate at full resolution is 3 frames per second (fps) and at VGA - 30 fps. It defines how many times per second the end-effector's position could be estimated.

One of its edges was chosen as a reference direction for the displacements of the scanning system. In this way, it appears parallel to one of the camera's directions. Therefore, the camera pixels form an orthogonal coordinate system. The scale of this coordinate system could be estimated by means of the translational stage. In general, the translational stage is set to perform a certain movement, the displacement is than measured by DIC in pixels and thus, a correspondence between the real and the image displacements is established. This procedure is usually called image space calibration. It should be considered, that the image space calibration should be performed over the whole imaging space in case of poor optical lenses, having large aberrations. In the realized experimental setup, a high quality lens was used and it has been found that one-pixel displacement in the image space corresponds to $0.268 \mu \mathrm{m}$ 
displacement in the end-effector's working space. The estimated error for this setup has been proven to be $0.055 \mu \mathrm{m}$.

Subsequently, on each piezo actuator has been applied a voltage from 0 to $100 \mathrm{~V}$ with step of $5 \mathrm{~V}$. After each voltage change, the end-effector position has been measured by DIC. In this way, both axes of the scanning system were defined and the working range estimated. In Fig. 6, it could be seen that the working range along $\mathrm{X}$ and $\mathrm{Y}$ directions is $22 \mu \mathrm{m}$ and the angle between them is $110^{\circ} \mathrm{C}$, which can be calculated from the equations of both linear interpolation curves.

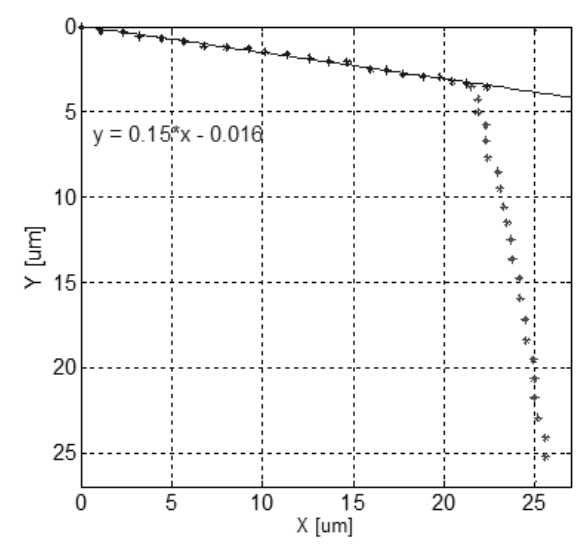

(a)

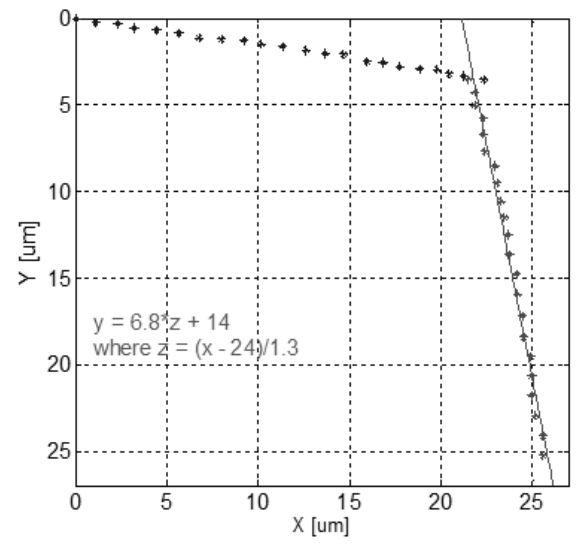

(b)

Fig. 6. Main axes and working range of the prototyped scanning system

All measured displacements depicted by starlets accumulated in a single image are shown in Fig. 7. Each starlet represents the end effector's position in the image plane estimated after each voltage change.

Simultaneous voltage measurements from the integrated MEMS position sensors have been acquired, as they are shown in Fig. 8. There is a liner correlation between the measured coordinates of the end-effector and MEMS position sensors' data. The coefficients of the interpolation equations, presented in Fig. 8, allow establishing one to one correlation between the value of MEMS sensors' output voltage and the coordinates of the end-effector. 


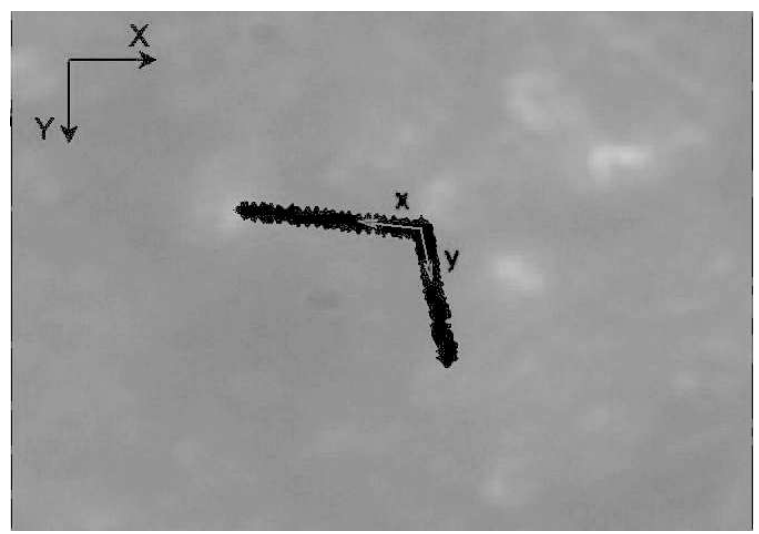

Fig. 7. Picture of the accumulated displacements of the end-effector during the voltage change

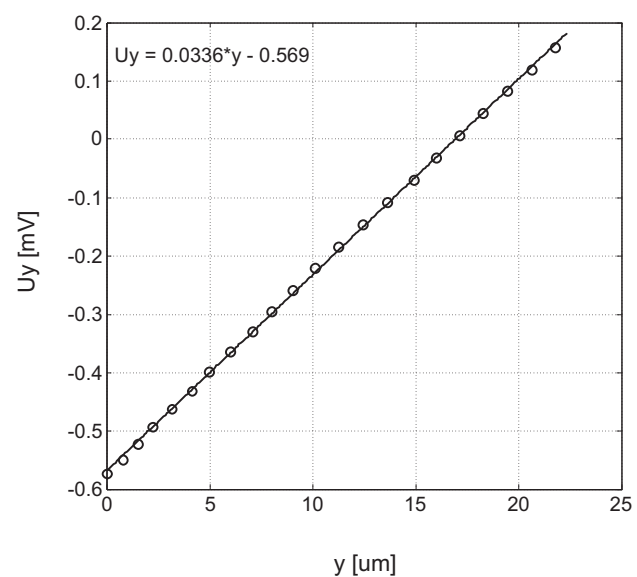

(a)

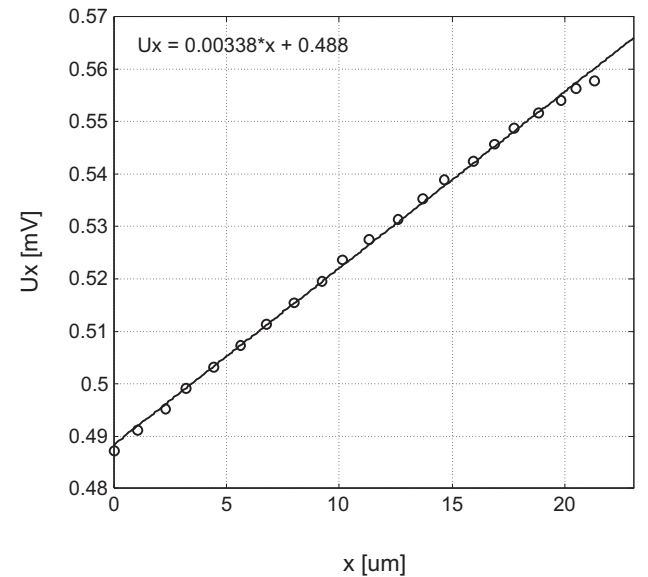

(b)

Fig. 8. MEMS measured values vs end-effector positions along both axes: a) $\mathrm{Y}$ and b) $\mathrm{X}$

\section{Conclusions}

In the work presented in this paper, a study of a mechatronic scanning system for application in the microbiology, microelectronics research, chemistry, etc. is presented. Integrated silicon MEMS sensors are used for monitoring the displacements in the scanning system. The developed silicon MEMS sensors with sidewall embedded piezoresistors possess a number of key advantages such 
as high sensitivity, better scaling characteristics, low noise and extremely low temperature dependence. In this work, the design of 2D scanning system with a travel range of $22 \times 22 \mu \mathrm{m}^{2}$ is presented. This system includes a Compliant Transmission Mechanism, designed as a complex elastic mechanism with four parallelograms incorporated. Stack multilayer piezo actuators are chosen as actuators of the system, because of their smooth motion, high accuracy, and quick response.

CAD model and FEA simulations of the elastic transmission mechanisms are carried out. A pre-stressing of the mechanical system is provided, in order to eliminate the backlash and improve the performance of the piezoactuators. The simulations of parallelogram elastic mechanism show linear displacement and symmetrical cross coupling between both axes.

A prototype of the scanning system, based on the developed CAD model, is manufactured. An experimental set-up for testing the scanning system prototype is developed, using an optical system and correlation technique for digital image processing. Experimental investigations of a prototyped scanning system have been presented. As a result of the experiment, both axes of the scanning system were defined and the working range along $\mathrm{X}$ and $\mathrm{Y}$ directions of $22 \mu \mathrm{m}$ were estimated. Simultaneous voltage measurements from the integrated MEMS have been acquired. They established liner correspondence between the measured coordinates of the end-effector and MEMS' data. The coefficients of the interpolation equations characterize one to one correspondence between the MEMS' values and coordinates of the end-effector.

Furthermore, investigations and experiments on 3D scanning are under consideration. Design and manufacturing of scanning system additional stage is going to be performed and a study of three axes displacements to be carried out. A control system based on MEMS sensors feedback is going to be developed.

\section{REFERENCES}

[1] Fatikow, S., U. Rembold. Microsystem Technology and Microrobotics, New York, Berlin Heidelberg, Springer-Verlag, 1997.

[2] Pernette, E., S. Henein, I. Magnani, R. Clavel. Design of Parallel Robots in Microrobotics. Robotica, 15 (1997), 417-420.

[3] Kasper, R., W. Heinemann, A. Wagner. Modelling and Control for Piezoelectric Actuators for High Speed Applications, Proceedings of $4^{\text {th }}$ Inl. Conf. on Motion and Vibration Control, 1 (1998), ETH Zürich, 231-236. 
[4] LI, Y., Q. Xu. Kinematic Design of a Novel 3-DoF Compliant Parallel Manipulator for Nanomanipulation, IEEE/ASME Int. Conf. on Adv. Intelligent Mechatronics, USA, Monterey, 2005, 24-25 July, 93-98.

[5] Yao, Q., J. Dong, P. M. Ferreira. A Novel Parallel-kinematics Mechanisms for Integrated, Multi-axis Nanopositioning: Part 1. Kinematics and Design for Fabrication. Precision Engineering, 32 (2008), No. 1, 7-19.

[6] Prusak, D., T. Uhl. Novel Type of Hybrid 3-DOF Micromanipulator with Piezoelectric Actuators - Mechanical Construction and Simulations. Solid State Phenomena, 147-149 (2009), 25-30.

[7] Yong, Y. K., T.-F. Lu. The Effect of the Accuracies of Flexure Hinge Equation on the Output Compliances of Planar Micro-motion Stages. Mechanism Machine Theory, 43 (2008), 347-363.

[8] Yong, Y. K., T.-F. Lu. Kinetostatic Modelling of 3-RRR Compliant Micromotion Stages with Flexure Hinges. Mechanism Machine Theory, 44 (2009), No. 6, 1156-1175.

[9] Chu, Ch.-L., Sh.-H. Fan. A Novel Long- travel Piezoelectric- driven Linear Nanopositioning Stage. Precision Engineering, 30 (2006), 85-95.

[10] Gao, P., S.-M. Swei. A Six-degree-of-freedom Micro-manipulator Based on Piezoelectric Translator. Nanotechnology, 10 (1999), 447-452.

[11] Kasper, R., M. AL-Wahab. Mechanically Structured Piezoelectric Actuators, Proceedings of $9^{\text {th }}$ International Conference on New Actuators - ACTUATOR 2004, Germany, Bremen, 2004, 68-71.

[12] Ionescu, Fl., K. Kostadinov. Piezo Actuated Micro Robot for Micro and Nano Manipulations. ARA Journal, 2000-2002 (2002), No. 25-27, Montréal, 98-103.

[13] Pashkevich, A., D. Chablat, P. Wenger. Stiffness Analysis of Overconstrained Parallel Manipulators. Mechanism Machine Theory, 44 (2009), No. 5, 966-982.

[14] Mohammed, A., W. A. Moussa, E. Lou. High-Performance Piezoresistive MEMS Strain Sensor with Low Thermal Sensitivity. Sensors, 11 (2011), 18191846; DOI: $10.3390 / \mathrm{s} 110201819$.

[15] Cao, L., T. Kim, S. Mantell, D. Polla. Simulation and Fabrication of Piezoresistive Membrane Type MEMS Strain Sensors. - Sensors and Actuators A: Physical, 80 (2000), 273-279.

[16] Han, B., J. Ou. Embedded Piezoresistive Cement-based Stress/strain Sensor. Sensors and Actuators A: Physical, 138 (2007), 294-298.

[17] Kumar, Sh., K. P. Venkatesh, S. S. Baskar, S. P. Madhavi. System Integration Design in MEMS-A Case Study of Micromachined Load Cell. Sadhana, 34 (2009), No. 4, 663-675.

[18] Stavrov, V., V. Todorov, A. Shulev, C. Hardalov. MEMS Sensors for mm-Range Displacement Measurements with Sub-nm-Resolution, CD Proc. of SPIE Conf. Microtechn., France, Grenoble, v. 8763 87632G-1-6A, 2013. 
[19] Todorov, V., G. Stavreva, V. Stavrov. Contact Mode MEMS Position Sensors with Piezoresistive Detection, Proc. XXVIII EUROSENSORS, Italia, Brescia, Procedia Engineering, Volume 87, doi:10.1016/j.proeng.2014.11.371, 2014, $1156-1159$.

[20] Stavrov, V., A. Shulev, D. Chakarov, G. Stavreva. Force Monitoring Transducers with More than 100,000 Scale Intervals, CD Proc. of SPIE Conf. Microtechn., Spain, Barcelona, v. 9517 95171Q-1-6, 2015.

[21] Kostadinov, K., D. Chakarov., T., Tiankov, A. Shulev. A Robot for Cell Injection: Modelling, Design and Experimental Validation. International Journal of Intelligent Mechatronics and Robotics, 3 (2013), No. 3, 77-98. 